\title{
Confinement Effects on Chain and Glass Dynamics in Immiscible Polymer Blends
}

\author{
Chen-Yang Liu, ${ }^{* \dagger}{ }^{\dagger}$ Baoqing Zhang, ${ }^{\dagger}$ Jiasong He, ${ }^{\dagger}$ Roland Keunings, ${ }^{*}$ and Christian Bailly ${ }^{\S}$ \\ ${ }^{\dagger}$ Beijing National Laboratory for Molecular Sciences, CAS Key Laboratory of Engineering Plastics, \\ Joint Laboratory of Polymer Science and Materials, Institute of Chemistry, Chinese Academy of Sciences, \\ Beijing 100080, China, ${ }^{\star}$ CESAME, Université catholique de Louvain, B-1348 Louvain-La-Neuve, Belgium, and \\ ${ }^{\S}$ Unité de Chimie et de Physique des Hauts Polymères, Université catholique de Louvain, B-1348 \\ Louvain-La-Neuve, Belgium
}

Received June 30, 2009; Revised Manuscript Received August 16, 2009

Dynamics of two-component polymer systems such as blends, ${ }^{1}$ copolymers, ${ }^{2}$ and nanocomposites, ${ }^{3}$ are complicated and not well understood at a fundamental level. It is clear from experimental evidence that the effect of one component on the dynamics of the second is nontrivial. ${ }^{4-7}$ For example, in contrast with wellentangled homopolymer melts, ${ }^{8}$ miscible polymer blends often show a dramatic failure of time-temperature superposition (tTS) principle, ${ }^{4,5}$ and very broad glass transitions are typically observed. ${ }^{7}$ It is generally accepted that these macroscopic observations are a result of distinct dynamics of the blends components, since each component experiences a different local friction from that of the pure polymer. Among the various models that attempt to predict miscible blend component dynamics, the self-concentration model proposed by Lodge and McLeish ${ }^{9}$ has received considerable attention recently. On the other hand, most investigations on immiscible blends have only focused on droplet breakup dynamics, but their molecular dynamics have received very little attention. We have designed a series of immiscible model blends wherein a small fraction of "probe" chains is dispersed in a very high-molecular weight (MW) phase-separated matrix, as a heretofore uninvestigated model system, in order to examine the impact of confinement from the matrix on the terminal and glass dynamics of the probe. Current theories ${ }^{10}$ suggest that confinement effects are expected when length scales are below 10 times the mean-square end-to-end distance $\left\langle R^{2}\right\rangle_{0}{ }^{1 / 2}$ usually ranging from 10 to $30 \mathrm{~nm}$. However, we have observed a strong confinement effect for micrometer-size dispersed droplets, which is much larger than this estimated critical dimension $\left(10\left\langle R^{2}\right\rangle_{0}{ }^{1 / 2}\right)$.

Narrow-disperse linear 1,4-polybutadiene (1,4 addition $>90 \%$, referred to as PBD in this work), 1,4-polyisoprene (1,4 addition $>90 \%, P I)$, and 1,2-polybutadiene (1,2 addition $>85 \%$, or polyvinylethylene, PVE) were purchased from Polymer Source, Inc. Molecular characterization of the samples is provided in Table 1. Very high-MW PBD and PI were used as the matrix for the immiscible blends and are hereafter denoted as PBD-matrix and PI-matrix, respectively. Three immiscible blends, PBD/PImatrix, PI/PBD-matrix and PVE/PI-matrix were investigated in this paper. Blends of $10 \mathrm{wt} \%$ low-MW and $90 \mathrm{wt} \%$ high-MW matrix components were mixed with $0.2 \% \mathrm{w} / \mathrm{w}$ Ciba IRGANOX B215 antioxidant, dissolved in excess toluene and then cast into film. The size of dispersed droplets was at the micrometer scale. ${ }^{12}$ Linear viscoelastic (LVE) properties of the binary blends and the pure polymers were measured using a TA ARES rheometer

\footnotetext{
*Corresponding author. E-mail: liucy@iccas.ac.cn.
}

with $8 \mathrm{~mm}$-diameter parallel-plate geometry. The details of LVE measurements have been described before. ${ }^{13,14}$

Figure 1 shows a pseudomaster curve (symbols) of the storage and loss moduli ( $G^{\prime}$ and $G^{\prime \prime}$, respectively) for the PBD/ PI-matrix blend as a representative of all the tested systems. The master curve of the high-MW PI-matrix is included (lines) for reference. In order to obtain the pseudomaster curve of this immiscible blend, the WLF (Williams-Landel-Ferry) shift factors of the pure PI-matrix were used because PI-matrix is the continuous phase. The two phases are expected to retain their individual temperature-dependent rheology, so the time-temperature superposition will fail. Indeed, the intermediate frequency $G^{\prime \prime}$ peaks corresponding to the terminal relaxation of the dispersed PBD phase appear at different positions in the pseudomaster curve, although a good overlap is observed at low and high frequencies (dominated by the PI-matrix signal). Furthermore, PBD peaks measured at lower temperature are shifted toward higher frequencies, since the shift factors of the pure PI-matrix have been used to shift the blend data and the PI-matrix has stronger temperature-dependent dynamics than PBD has because the former has a higher glass transition temperature $(-63$ vs $\left.-99{ }^{\circ} \mathrm{C}\right) .{ }^{15}$

For narrow-disperse polymers, such as these used here, the frequency of the terminal $G^{\prime \prime}$ peak, $\omega_{\max },{ }^{16}$ is connected to the

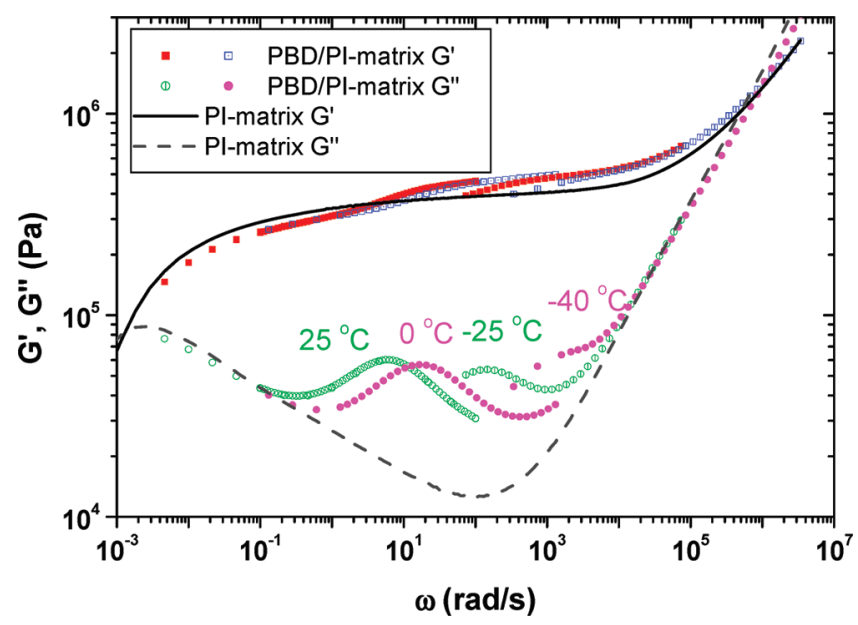

Figure 1. "Master curve" (symbols) of the immiscible PBD/PI-matrix $(10 / 90)$ blend obtained by using the shift factors of the PI-matrix. Failure of time-temperature superposition is observed because of different temperature-dependences of the components dynamics. Also plotted for reference is the master curve of the PI-matrix at $25^{\circ} \mathrm{C}$. 

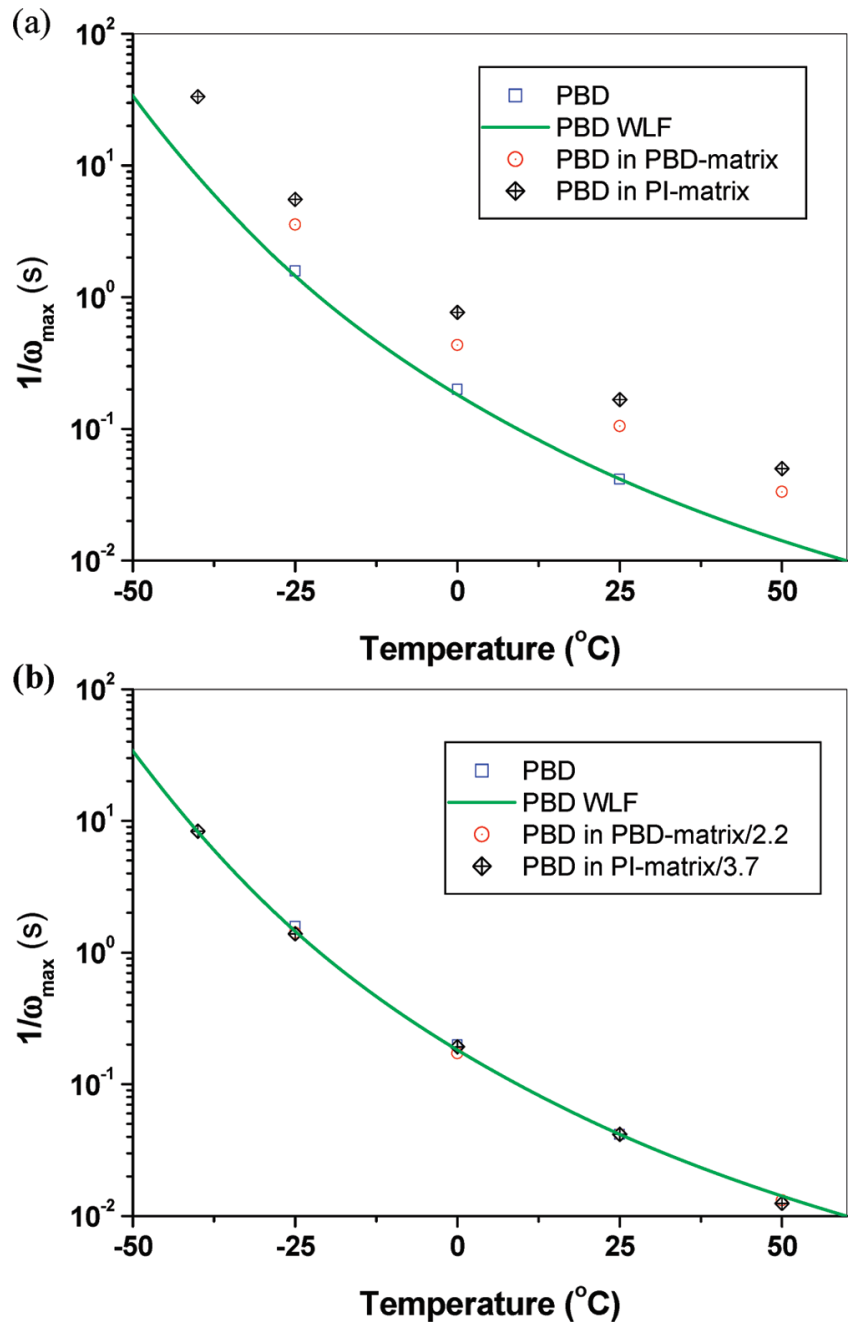

Figure 2. (a) Temperature dependence of terminal relaxation times for the pure $\mathrm{PBD}$, the $10 \% \mathrm{PBD}$ probe in the miscible $\mathrm{PBD} / \mathrm{PBD}$-matrix blend $^{14}$ and in the immiscible PBD/PI-matrix blend. The curve represents the WLF fitting of LVE data of the pure PBD. (b) Terminal relaxation times for the PBD probe in the PBD matrix ${ }^{14}$ and in the PI-matrix are vertically shifted to overlap with data of the pure PBD.

terminal relaxation time $\tau_{d}$ by the simple relation: ${ }^{17}$

$$
\tau_{d}=1 / \omega_{\max }
$$

It is worth noting that the PI-matrix is in the pseudorubbery state (the plateau region) in the frequency range where the terminal relaxation peak of the PBD phase occurs, as shown in Figure 1, since the terminal relaxation time of the PI-matrix is more than 1000 times longer than that of the PBD phase. Temperature dependence of $\tau_{d}$ for the dispersed PBD phase in the PI-matrix is presented in Figure 2a. Also plotted for reference are terminal relaxation times of pure PBD and the WLF fit of the LVE data, which extends the temperature range for $\tau_{d}$. Recently, we also investigated the relaxation dynamics of probe chains in a model environment of quasi-permanent entanglements, by analyzing a mixture of $10 \%$ short chains and $90 \%$ long chains PBD. ${ }^{14}$ Terminal relaxation times for this system are also presented in Figure 2a.

Now for the first time, we can compare the relaxation dynamics of the same narrow-disperse PBD in three different environments: (1) an entanglement network consisting of identical PBD chains; (2) a quasi-permanent entanglement network consisting of the same polymer ${ }^{14}$ but with more than 100 times slower terminal relaxation (PBD-matrix); (3) an entanglement
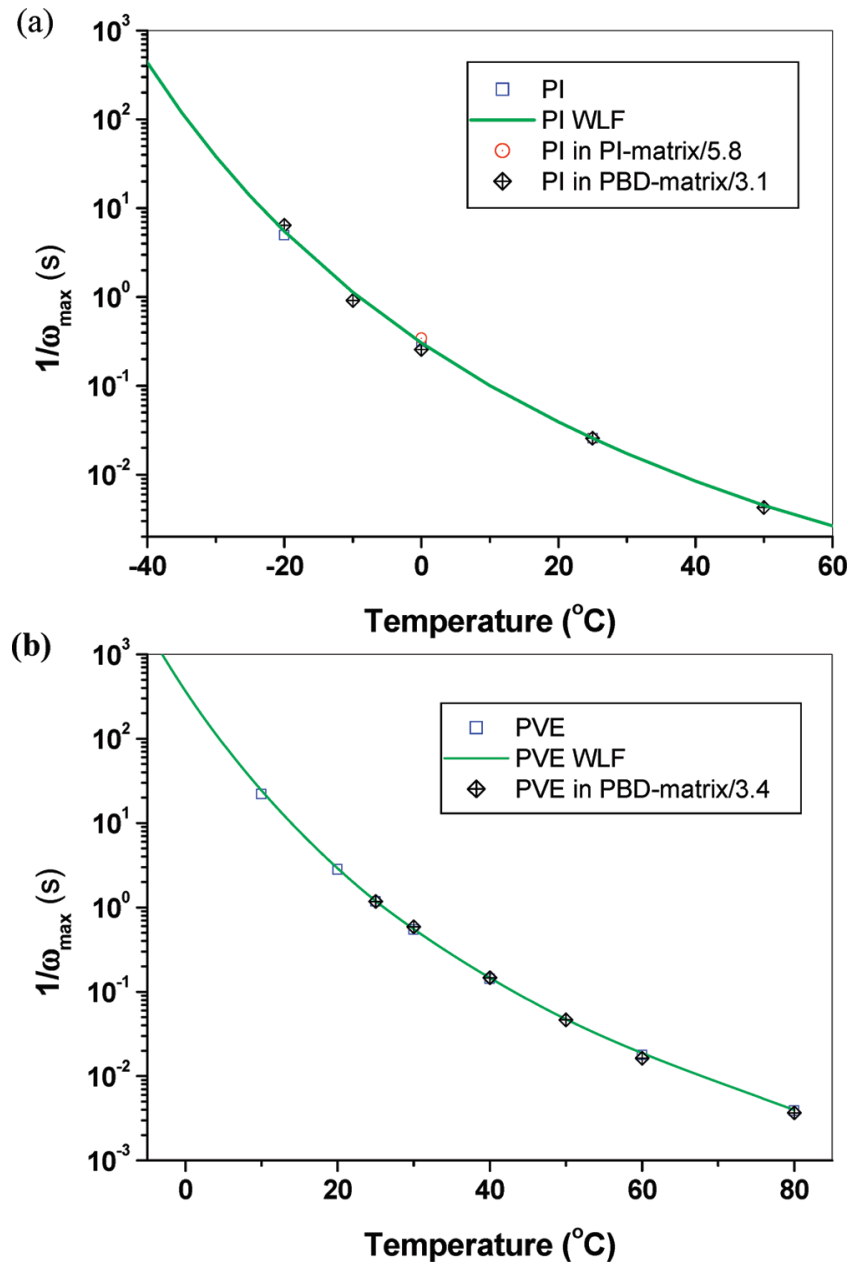

Figure 3. (a) Terminal relaxation times for the $10 \%$ PI probe in the PI/PI-matrix miscible blend ${ }^{14}$ and in the PI/PBD-matrix immiscible blend are vertically shifted to overlap with data of the pure PI. The curve represents the WLF fitting of LVE data of the pure PI. (b) Terminal relaxation times for the 10\% PVE probe in the PVE/ PBD-matrix immiscible blend are vertically shifted to overlap with data of the pure PVE. The curve represents the WLF fitting of LVE data of the pure PVE.

network consisting of micrometer-size dispersed PBD droplets ${ }^{12}$ in a high-MW PI-matrix. The remarkable result is that the PBD dynamics in these three different environments have the same temperature-dependence, but are significantly slower in the miscible PBD/PBD-matrix and immiscible PBD/PI-matrix blends than in the narrow-disperse PBD. This can be clearly seen in Figure 2b, where $\tau_{d}$ for PBD in the PBD-matrix and in the PI-matrix are vertically shifted down to overlap with data of neat PBD. Their retardation factors ${ }^{18}$ are 2.2 and 3.7, respectively.

Different mechanisms presumably lead to the observed retardation. It is accepted that the tube motion mechanism is suppressed in the quasi-permanent entanglement PBD network, explaining the slower terminal relaxation in the $\mathrm{PBD} / \mathrm{PBD}$ matrix blend. ${ }^{14}$ However, retardation of chain dynamics for a dispersed PBD phase trapped in an immiscible PI-matrix is reported here for the first time. This observation can be linked to dynamic confinement effects recently observed by Kimmich's group, ${ }^{19,20}$ with the help of field-cycling NMR relaxometry on perfluoropolyether melts confined in micrometer thick layers of polyimide. The authors found that chain dynamics under such moderate confinement conditions $(0.8-1.6 \mu \mathrm{m}$ thick layers between polyimide films) were perceptibly slower than observed in the bulk material with a shift factor about 2, and the 
Table 1. Molecular Characterization and Relaxation Results

\begin{tabular}{lccccccc}
\hline \multicolumn{1}{c}{ sample } & $M_{\mathrm{w}}(\mathrm{kg} / \mathrm{mol})$ & $M_{\mathrm{w}} / M_{\mathrm{n}}$ & $\left\langle R^{2}\right\rangle_{0}{ }^{1 / 2}(\mathrm{~nm})^{a}$ & retardation factors & $T_{\mathrm{g}}\left({ }^{\circ} \mathrm{C}\right)$ & $T_{\mathrm{g}}$ in matrix $\left({ }^{\circ} \mathrm{C}\right)$ & matrix \\
\hline PBD & 98.8 & 1.03 & 29.3 & 3.7 & -98 & $-67(-65)^{b}$ & $-50^{c}$ \\
PI & 81.8 & 1.07 & 22.1 & 3.1 & $-20(-17)^{b}$ & $-5^{c}$ & PBD-matrix \\
PVE & 51.4 & 1.06 & 18.5 & 3.4 & -100 & PBD-matrix \\
PBD-matrix & 1240 & 1.13 & 104 & & -62 & \\
PI-matrix & 1310 & 1.1 & 88.4 & &
\end{tabular}

${ }^{a}$ Calculated by using data of chain dimensions in ref $11 .{ }^{b}$ The temperature at which there is a $G^{\prime} / G^{\prime \prime}$ cross-point at $1 \mathrm{rad} / \mathrm{s} .{ }^{c}$ The temperature at which there is a local maximum of $G^{\prime \prime}$ at $1 \mathrm{rad} / \mathrm{s}$.
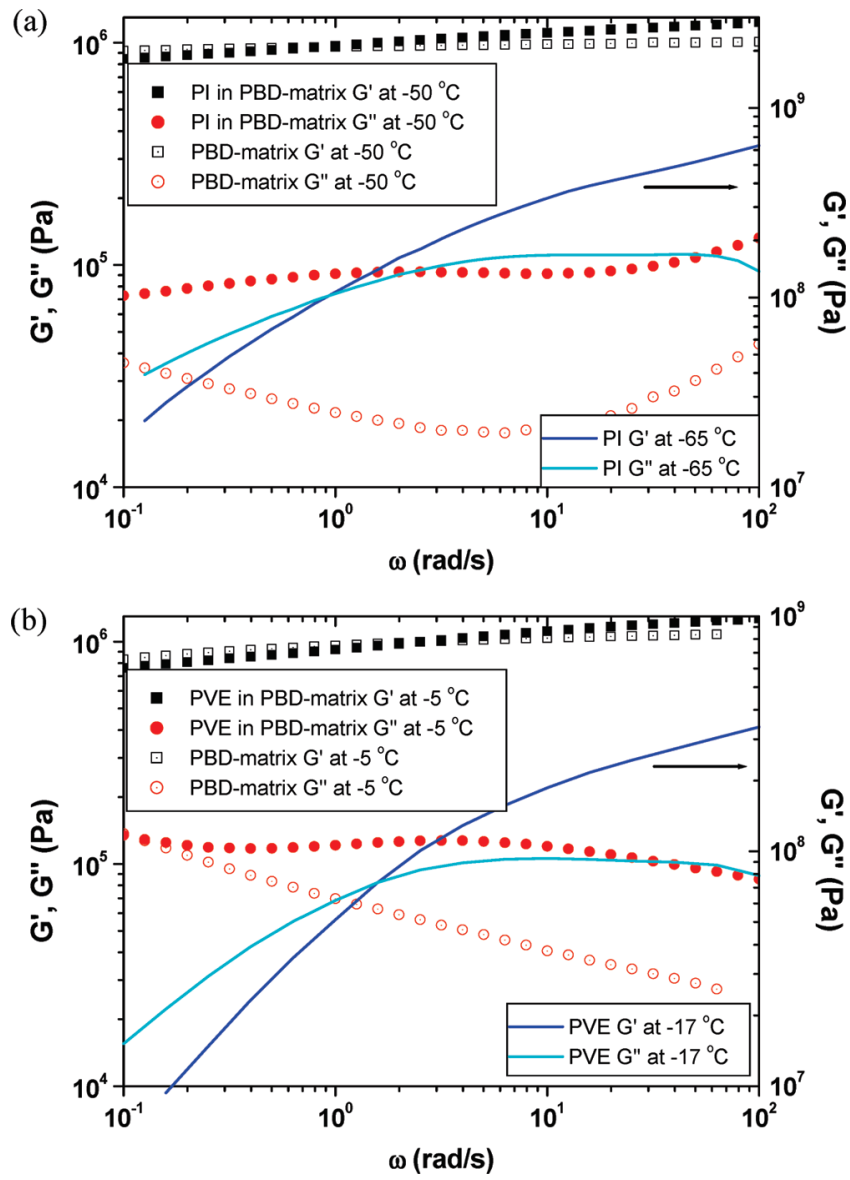

Figure 4. (a) High- $T_{\mathrm{g}}$ component $\mathrm{PI}$ in the immiscible PI/PBD-matrix blend. This shows a glass relaxation $\left(G^{\prime \prime}\right.$ peak around $\left.2 \mathrm{rad} / \mathrm{s}\right)$ at $-50^{\circ} \mathrm{C}$. The LVE data of the PBD-matrix is plotted as a reference. The pure PI (right Y-axis) shows a glass relaxation at $-65^{\circ} \mathrm{C}$ (DSC-measured $T_{\mathrm{g}}$ at $-67{ }^{\circ} \mathrm{C}$ ). (b)High- $T_{\mathrm{g}}$ component PVE in the immiscible PVE/ PBD-matrix blend. This shows a glass relaxation $\left(G^{\prime \prime}\right.$ peak around 3 $\mathrm{rad} / \mathrm{s}$ ) at $-5{ }^{\circ} \mathrm{C}$. The LVE data of the PBD-matrix is plotted as a reference. The pure PVE (right Y-axis) shows a glass relaxation at $-17^{\circ} \mathrm{C}\left(\mathrm{DSC}\right.$-measured $T_{\mathrm{g}}$ at $\left.-20^{\circ} \mathrm{C}\right)$.

characteristic relaxation time increased with decreasing layer thickness. Although the confinement was one-dimensional (layers sandwiched between films) in their case and threedimensional (dispersed as droplets) in our immiscible PBD/PImatrix blend, the confinement for both cases is at the micrometer scale.

To check the universality of the dynamic confinement phenomenon, two other immiscible blends, PI/PBD-matrix and PVE/PBD-matrix, were prepared. Dynamic confinement effects were also observed in these two blends. Parts a and b of Figure 3 show that retardation factors are 3.1 and 3.4, respectively. Since retardation factors from 3.1 to 3.7 for three different dispersed blend systems are quite consistent with each other, the confinement effects are not expected to be due to some specific physicochemical effects.
In immiscible PI/PBD-matrix and PVE/PBD-matrix blend systems, the dispersed phases have higher $T_{\mathrm{g}}$ 's than that of the matrix, therefore the glass relaxation dynamics of the probe droplets can be investigated. The confinement effect on glass dynamics is indeed observed from LVE data. In parts a and b of Figure 4, the PI and PVE fractions in PBD-matrix show a $G^{\prime \prime}$ relaxation peak around $2 \mathrm{rad} / \mathrm{s}$ at $-50{ }^{\circ} \mathrm{C}$ and around $3 \mathrm{rad} / \mathrm{s}$ at $-5{ }^{\circ} \mathrm{C}$, respectively. This relaxation is ascribed to the glass transition of the PI and PVE dispersed phases. ${ }^{21}$ The DSCmeasured $T_{\mathrm{g}}$ of PI is about $-67{ }^{\circ} \mathrm{C}$ (Table 1 ). This is consistent with the rheological glass relaxation temperature. Indeed, Figure 4a shows that pure PI has a GPa-magnitude plateau and a $1 \mathrm{rad} / \mathrm{s} G^{\prime} / G^{\prime \prime}$ cross-point at $-65^{\circ} \mathrm{C}$. Similarly, Figure $4 \mathrm{~b}$ shows that PVE has a glass relaxation at $-17^{\circ} \mathrm{C}$, which is very close to its DSC-measured $T_{\mathrm{g}}$ of $-20^{\circ} \mathrm{C}$. Hence the $T_{\mathrm{g}}$ 's measured by LVE test for dispersed PI and PVE in the PBD-matrix increase by approximately $10{ }^{\circ} \mathrm{C}$ as compared with the bulk polymers. This is the first report of a $T_{\mathrm{g}}$ increase for a polymer dispersed at the micrometer scale in an entangled matrix. The closest case in literature is a $3{ }^{\circ} \mathrm{C}-T_{g}$ increase observed for PS in an immiscible polystyrene/high density polyethylene blend, ${ }^{22}$ where the confinement effect has been attributed to the physical state of PE semicrystalline domains.

In this paper, the observed increases of terminal relaxation time and glass transition temperature occur under mild confinement conditions, i.e. model systems consisting of a small fraction of short probe chains as dispersed droplets in a very high-MW matrix. The size of the dispersed phase is at the micrometer scale, which is much larger than the generally accepted confinement dimension of $10\left\langle R_{\mathrm{g}}{ }^{2}\right\rangle_{0}{ }^{1 / 2} \cdot{ }^{10,19,20}$ Since the retardation factors of the terminal relaxation time are quite consistent for these three distinct blends and the $T_{\mathrm{g}}$ increases for these two distinct blends are also very close, these confinement effects involved are not expected to be due to any specific physicochemical effect. The interface structure between two phases ${ }^{23}$ and the entanglement state of high-MW matrixes may play key roles for the dynamic confinement effects. We hope this paper will stimulate new theoretical simulation and experimental investigation to help resolve the uncovered issue.

Acknowledgment. This work is supported by National Natural Science Foundation of China (Grant No. 20874109), and the Chinese Academy of Sciences.

Supporting Information Available: Text giving additional experimental details for morphology of the immiscible blends, for the retardation of chain dynamics for a dispersed phase trapped in a heterogeneous entangled matrix, and for the glass transition of high- $T_{\mathrm{g}}$ dispersed phase with figures showing phase contrast micrographs, plots of LVE data, and plots of $G^{\prime}$ and $G^{\prime \prime}$ moduli. This material is available free of charge via the Internet at http://pubs.acs.org.

\section{References and Notes}

(1) Haley, J. C.; Lodge, T. P. J. Rheol. 2004, 48, 463.

(2) Fredrickson, G. H.; Bates, F. S. Annu. Rev. Mater. Sci. 1996, 26, 501. 
(3) Moniruzzaman, M.; Winey, K. I. Macromolecules 2006, 39, 5194.

(4) Colby, R. H. Polymer 1989, 30, 1275.

(5) Pathak, J. A.; Colby, R. H.; Floudas, G.; Jerome, R. Macromolecules 1999, 32, 2553.

(6) Haley, J. C.; Lodge, T. P.; He, Y. Y.; Ediger, M. D.; von Meerwall, E. D.; Mijovic, J. Macromolecules 2003, 36, 6142.

(7) Lodge, T. P.; Wood, E. B.; Haley, J. C. J. Polym. Sci., Polym. Phys. Ed. 2006, 44, 756.

(8) Heymans, N. Macromolecules 2000,33, 4226. The critical molecular weight of some polymers can be different at different temperatures, so the tTS principle only holds for well-entangled homopolymer melts.

(9) Lodge, T. P.; McLeish, T. C. B. Macromolecules 2000, 33, 5278.

(10) Fatkullin, N.; Kimmich, R.; Fischer, E.; Mattea, C.; Beginn, U.; Kroutieva, M. New J. Phys. 2004, 6, 46. The length "10 times the mean-square end-to-end distance" should be interpreted as a low limit range where the confinement is expected to be effective.

(11) Fetters, L. J.; Lohse, D. J.; Richter, D.; Witten, T. A.; Zirkel, A. Macromolecules 1994, 27, 4639.

(12) Morphologies of these immiscible blends studied are shown in the Supporting Information. Although Jeon et al. [ Jeon, H. S.; Nakatani, A. I.; Han, C. C.; Colby, R. H. Macromolecules 2000, $33,9732]$ reported a critical solution temperature of $61.5^{\circ} \mathrm{C}$ for a PBD/ PI blend, the phase behavior of a binary blend is a function of the molecular weight. See:Roovers, J.; Toporowski, P. M. Macromolecules 1992, 25, 1096. These blends studied in our work have a broad two-phase region, since the matrixes used have very high-molecular weights.

(13) Liu, C. Y.; He, J. S.; Keunings, R.; Bailly, C. Polymer 2006, 47, 4461.

(14) Liu, C. Y.; Halasa, A. F.; Keunings, R.; Bailly, C. Macromolecules 2006, 39, 7415.

(15) Liu, C. Y.; He, J. S.; Keunings, R.; Bailly, C. Macromolecules 2006, $39,8867$.

(16) The $\omega_{\max }$ at $-40{ }^{\circ} \mathrm{C}$ for the PBD component was estimated from the terminal $G^{\prime \prime}$ peak obtained by subtracting the $G^{\prime \prime}$ data of the pure PI-matrix from the $G^{\prime \prime}$ data of the blend.

(17) Graessley, W. W. J. Polym. Sci., Polym. Phys. Ed. 1980, 18, 27.

(18) The retardation factor is the ratio of the $\tau_{d}$ under the confinement condition to the $\tau_{d}$ of the pure polymer.

(19) Kausik, R.; Mattea, C.; Fatkullin, N.; Kimmich, R. J. Chem. Phys. 2006, 124, 114903 .

(20) Kausik, R.; Mattea, C.; Fatkullin, N.; Kimmich, R. Eur. Phys. J. E 2007, 141, 235.

(21) See Supporting Information.

(22) Thirtha, V.; Lehman, R.; Nosker, T. Polymer 2006, 47, 5392.

(23) Hashimoto, T.; Takenaka, M.; Jinnai, H. J. Appl. Crystallogr. 1991, 24, 457The interface structure in the spinodal decomposition for the PI/PBD blend was evaluated by a time-resolved light scattering, and the equilibrium thickness of phase-phase interfaces is about $250 \mathrm{~nm}$. 